\title{
The effect of cutting head vibrations on the surfaces generated by waterjet cutting
}

\author{
M. Monno, C. Ravasio - Politecnico di Milano, Italy
}

\section{$\underline{\text { Abstract }}$}

After a first period in which the research has been focused on the optimisation of the process parameters, the attention is now focused on aspects that were usually neglected. However, they are very important in order to understand the physics of the waterjet / abrasive waterjet cutting process and to improve the cutting quality.

Particularly, it has been demonstrated that, in the pure waterjet cutting (and in the abrasive waterjet cutting too), there are irregularities, called striations, along the generated surface. The striation formation depends mainly on the jet instability caused by vibrations during the cutting process. Vibration signals have been measured whilst varying the cutting conditions. A model has been studied which estimates the mean spacing and the frequency of the striations, as a function of the period and the amplitude of the jet vibration. This model has been completely validated through measurements of plasticine surfaces generated by waterjet cutting.

\section{$\underline{\text { Keywords }}$}

Vibrations, cutting quality

\section{Introduction}

The Waterjet and Abrasive Waterjet technology is the object of a concentrated research. This research area is oriented to understand the material removal mechanisms and to 
optimise the process parameters (fluid dynamical and technological) of various applications.

The optimisation of the process parameters is especially difficult because of their instability during the process. Many authors have inquired into the physical-mechanical aspects of the interaction between the jet and the mechanical properties of the material and they developed models and different interpretations. However, the existing models, mostly, try to describe the cutting mechanism only for AWJ technology [1].

In AWJ cutting, the surface is characterised of two different zones: the upper one shows a random roughness profile while the lower one is characterised of a pronounced undulation (striations). The striations are formed when the ratio between the available energy of the jet and required energy of the destruction becomes comparatively small. Thus the striations can be reduced by the reduction of the traverse rate. This, however, damages the productivity and, what is the most important, does not eliminate the striations.

Research works have highlighted that the striations are caused by internal and external mechanisms of the AWJ cutting process [2-5].

The traditional interpretation of the mechanism of striation formation in AWJ cutting [6] claims that striations appear beyond a certain depth and they are seen especially in cutting of very thick materials. This result is in contrast with what has been found for WJ technology. In fact, along the whole waterjet cutting surfaces, there are irregularities (striations) with dimensions comparable to the jet diameter. Therefore, the AWJ models do not explain the mechanism of striation formation in WJ cutting. 


\section{State of the art}

Hashish founds the responsible causes of the striation generation in AWJ cutting and he subdivides them into [7]:

- process causes: the striation are the result of the removal material mechanism;

- causes involved in the process parameters control: the instability of the process parameters (pressure, abrasive flow rate, feed rate, etc.) is responsible for the striation formation;

- causes involved in the support equipment: the vibrations of the piece and/or the nozzle during the cutting process cause the striation formation.

Hashish [7] discovers an equation that connects the amplitude of waviness $\left(\mathrm{R}_{\mathrm{w}}\right)$ to the diameter of the jet $\left(d_{n}\right)$, the traverse rate $(u)$, the penetration depth $(h)$, the cutting wear depth $\left(h_{c}\right)$, the jet velocity $\left(V_{j}\right)$, the abrasive mass flow rate $\left(m_{a}\right)$ and the specific energy for deformation wear mode $(\varepsilon)$ :

$$
\frac{2 \mathrm{R}_{\mathrm{w}}}{\mathrm{d}_{\mathrm{n}}}=1-\sqrt{1-\left(\frac{\pi}{4}\right)^{2} \cdot\left[\frac{\mathrm{d}_{\mathrm{n}}\left(\mathrm{h}-\mathrm{h}_{\mathrm{c}}\right) \mathrm{u}}{0.5 \mathrm{~m}_{\mathrm{a}} \mathrm{V}_{\mathrm{j}}^{2} / \varepsilon}\right]^{2}}
$$

If the material thickness is equal to or less than $h_{c}$ (cutting wear depth), then this equation cannot be used. In this case, the cut surface will be rough rather than being wavy. When the material thickness is less than $h_{d}$ (deformation wear depth) and greater than $h_{c}$, the surface will be wavy and the waviness can be predicted from the equation. In accordance with this interpretation, in WJ cutting, striations should not exist or, however, should be in a limited zone and appear beyond a certain depth $\left(\mathrm{h}_{\mathrm{c}}\right)$. In fact, in this case, the materials that can be cut have a very low resistance to the penetration: when $\varepsilon$ decreases, $\mathrm{R}_{\mathrm{w}}$ decreases too. 
A different approach was made by Louis, Meier e Guo [8]: their attention has been focused on the influence of the physics of the process on the cutting surface structure. They found that:

- while the surface roughness is caused by the interaction between single particles and material and the striation with short wave length is formed by the overlapping of penetrations of the adjacent jets, the inclination and the unsteady oscillation of the jet lead to the formation of the grooves with large wave length.

- with increasing depth of measuring position the spectral distribution is concentrated in a narrow range of frequency, which demonstrates a dominant harmonic component in the surface profiles. This dominant component is more and more marked when measuring in increasing depth. The highest peaks of power spectrum are found approximately at the same frequency for different depth of cut in the region of rough cutting;

- the frequency and the amplitude of the dominant wave component as well as the surface profile depend strongly on the cutting parameters and the thickness of the workpiece. A variation of the diameter of the jet, which is related to the diameter of the focus, influences substantially the characteristics of the dominant wave component. With increasing focus diameters the significant signal of power spectrum is displaced to lower frequencies (larger wave length).

In [9], it has been deduced that the surface generated by AWJ cutting suffers strongly from the effects of the cutting head movement: the surface striations can be directly attributed to the cutting head vibrations. Particularly, at different feed rate, the power spectrums of the vibrations, measured on the cutting head, have been correlated with the harmonics of the power spectrums of the roughness profiles of the cut surface. The 
surface morphology does not show two distinct zones but the striations begin at the top of the cut and progressively worsen as the depth of the cut increases due to vibration and the energy decrease of the abrasive jet [10].

The suspicion that the vibrations are the main cause of the striation formation has found validation in WJ cuts on materials at low density like rubber or plasticine (Figure 1). The striation on the cut surfaces has confirmed the existence of a vibratory component in the jet. In fact, the striations appear from the top edge of the material and their dimension is comparable with the jet diameter.

In [11] a phenomenological model of striation formation, based on variations in the geometry of the cut front, has been developed and a range of moving steps on the cut front has been confirmed by the use of high speed video recording of the cut front when cutting transparent materials.

In [12], the striation formation is believed to be caused by the wavy abrasive particle kinetic energy distribution related to the cut surface. All factors which have an effect on this kinetic energy magnitude and its distribution related to the cut surface must affect the striation patterns and result in striation irregularities. Therefore the causes of striation formation have been described in terms of the internal effect which is wavy abrasive particle kinetic energy distribution and external effects such as: fluctuations or unsteadiness of the AWJ process parameters (such as traverse speed, pressure and abrasive flow rate), vibration of the workpiece and nozzle while cutting, vibration due to the nozzle traverse system.

In general, the vibrations during the WJ cutting process involve every component of the system and have effects on the cutting quality [13-14]. 
In this paper, the presence and the magnitude of the vibrations in the WJ cutting process and their effect on the cutting quality have been studied.

\section{Experimental procedure}

The vibrations can be subdivided in external, or exogenous, and in intrinsic, or endogenous. The vibrations on the workpiece caused by the jet, which interacts in air with the workpiece, the equipment and the water in the catcher, are exogenous. The inherent vibrations of the jet caused by the cutting head vibrations, by the movement system, by the intensifier system and by the nozzle, are endogenous.

Every source of vibration has been investigated and their magnitude and effects on surface quality of low-density materials, like rubber, have been quantified. In fact, the cut surface of very soft materials is affected by every noise phenomenon during the cutting.

\subsection{The exogenous vibrations}

The impact between high-speed jet and the water in the catcher generates the splashback phenomenon, i.e. heavy sprinklings of water. These sprinklings cause considerable vibrations in the whole machine and have an unfavourable effect on the WJ cutting quality. In fact, the water in the catcher dampens the residual energy of the jet after the cut and, during the process, surges strongly. It is possible to estimate these vibrations indirectly through the measure of the surface roughness.

In order to quantify the effect of these vibration, a suitable workpiece fixing system has been realised (Figure 2-b), able to dampen completely the residual energy of the jet after the cut and therefore able to prevent the sprinklings of water in the cutting area. 
Using this equipment, the quality surface, in term of roughness, should be better.

Two types of test have been carried out: in the first one, the standard fixing equipment has been used (case A); in the second one, the splash back damping equipment (case B). Rubber has been cut, in the both test (16 replications for every case), using a double acting intensifier system $(30 \mathrm{~kW})$ with the following process parameter:

- pressure: $350 \mathrm{MPa}$

- traverse rate: $200 \mathrm{~mm} / \mathrm{min}$

- nozzle diameter: $0.3 \mathrm{~mm}$

- $\quad$ stand off distance: $2 \mathrm{~mm}$

The synthetic roughness indexes of the generated surfaces have been measured through a contact-roughness-measuring machine at three different depth $(10,20,30 \mathrm{~mm})$ from the front edge of the test piece.

\subsection{The endogenous vibrations}

The mean spacing between the striations is comparable to the jet diameter $\left(d_{n}\right)$. This observation permits to think that the striation formation is due to the jet instability. However, a direct measure of the jet vibration is not feasible; the jet vibration is estimated indirectly, through the measure of the head vibrations in proximity of the jet exit, using a motion transducer (HBM W2ATK) and an accelerometer (PCB 308B) (Figure 3). Such indirect vibrations are not necessarily equivalent to the jet vibrations. In fact, the type of constraint between jet and cutting head is unknown. A reasonable assumption, due to the high speed jet, is that the constraint is rigid. In fact, at high speed (approximately $900 \mathrm{~m} / \mathrm{s}$ ), a coherent jet can be considered like a rigid body. 
This assumption permits to assume that the cutting head transmits its vibrations to the jet, and vice versa.

Figure 4 shows a numerical simulation that compares the theoretical WJ cutting with the one that assumes the cutting head transfers its vibrations to the jet.

The cutting head vibrations have been measured with open jet when the cutting head was still and in the other case when it was in motion. The motion does not cause new harmonics but amplifies the one that was obtained when the cutting head was still.

In the carried out tests, the cutting head vibration signal has been measured, with the jet open, at different level of wear of the nozzle. In particular, three tests at the same value of pressure (350 MPa) have been carried out:

- new nozzle, diameter $0,20 \mathrm{~mm}$

- $\quad$ new nozzle, diameter $0,30 \mathrm{~mm}$

- wear nozzle, nominal diameter $0,30 \mathrm{~mm}$.

These tests have confirmed that the most part of the vibrations is generated by the nozzle. In fact using a bigger nozzle, the vibration component has wider amplitude in the acquisition directions. Moreover, using a wear nozzle, the vibration becomes bigger in frequency and amplitude.

\section{Experimental results}

\subsection{Exogenous vibrations}

From the mean measures at different depth of the two tests (standard equipment and splash-back damping equipment), reported in Table 1, the following observations come out: 
- as the depth of measure increases, every parameter shows a growing trend, either the mean (Figure 5-a) or the dispersion (Figure 5-b);

- the surface quality using the splash back damping equipment (Case B) is better than that obtained using the standard equipment (Case A). At the same depth of measure, in fact, the Case B indicates a decrease in every parameter either in the mean or in the dispersion.

Particularly, all the synthetic roughness indexes show a trend like that in Figure 5.

The generated surfaces, using the splash-back damping equipment (Figure 2-b) have, on average, a lower roughness than those obtained using the standard equipment. In fact, the mean and the variance of the synthetic roughness indexes, at every depth of measure, decrease using a splash back damping system (Figure 5). The trend of the two curves is divergent, according to the effectiveness of the damping system, especially near the exit edge.

\subsection{Endogenous vibrations}

From the analysis of the cutting head vibration signal, it comes out that the oscillation amplitude is approximately $35 \mu \mathrm{m}$ and there is a frequency peak at $20 \mathrm{~Hz}$ (Figure 6).

Figure 7 shows the vibration power spectrums measured, respectively, in position $\mathrm{X}$ and Y (Figure 4-b) with the jet opens. It comes out that, as a function of the position of measure, the vibration amplitude increases while the frequency components are the same.

Figure 8 shows the vibration power spectrums, generated using the three types of nozzle:

- new nozzle, diameter $0,20 \mathrm{~mm}$ (a) 
- new nozzle, diameter $0,30 \mathrm{~mm}(\mathrm{~b})$

- wear nozzle, nominal diameter 0,30 $\mathrm{mm}(\mathrm{c})$.

Using a bigger nozzle, the vibration component has wider amplitude in the acquisition directions. Moreover, using a wear nozzle, the vibration becomes bigger in frequency and amplitude: in fact, when the jet comes across physical defects in the nozzle, it does not come off the walls with laminar motion, but generates some high turbulence zones that excite a vibration of the nozzle support.

Consequently, this will also cause jet instability, leading to a loss of energy and cutting efficiency; hence loss of cut quality.

\section{The geometric model}

The cutting of very soft materials with the lowest resistance to the jet penetration, like rubber, have shown the striation morphology on the separation surface even from the top edge of the material. This result, in addition to the vibration measurements carried out in several positions of the cutting head, has shown that the striations are caused by multiple vibration phenomenon generated during the cutting process. These are in accordance with the latest trends of research. Therefore, after the acknowledgement of the strong correlation between vibrations and surface striations, a geometric model has been developed, which describes the formation mechanism of the WJ cutting striations. The model, inspired from Hashish [7], assumes the hypothesis that the striation morphology is caused mainly by vibration phenomenon during the cutting process. Particularly, the model predicts, knowing the amplitude (A) and the frequency $\left(f_{v}\right)$ of the exogenous vibrations measured on the cutting head, the spatial frequency $(1 / \mathrm{X})$ and the amplitude $\left(\mathrm{R}_{\mathrm{w}}\right)$ of the surface striation (Figure 9). 
$\xi$ is a corrective geometric coefficient that considers that the periodical envelope of the circumferences has a lower sinusoidal amplitude.

The frequency components of the vibrations measured on two orthogonal axis are the same (Figure 7). Therefore, the motion of jet centre can be described as a composition of a translation motion and an uniform rectilinear motion: the jet axis describes on the cutting plan (x-y) an epicycloidal trajectory. The equations, that describe this motion, are:

$\left\{\begin{array}{l}x=A \cdot \cos \left(2 \pi \cdot f_{v} \cdot t\right)+u \cdot t \\ y=A \cdot \sin \left(2 \pi \cdot f_{v} \cdot t\right)\end{array}\right.$

where $f_{v}$ is the jet oscillation frequency, $u$ is the feed rate and $A$ is the oscillation amplitude.

Enveloping the side surface of the jet, whose centre follows the previous trajectory, striations can be obtained. The parametric equations of the family of circumferences, that have the centre making an epicycloidal motion, are:

$$
\left\{\begin{array}{l}
\mathrm{x}=\mathrm{A} \cdot \cos \left(2 \pi \cdot \mathrm{f}_{\mathrm{v}} \cdot \lambda\right)+\mathrm{u} \cdot \lambda+\frac{d_{j}}{2} \cos (t) \\
\mathrm{y}=\mathrm{A} \cdot \sin \left(2 \pi \cdot \mathrm{f}_{\mathrm{v}} \cdot \lambda\right)+\frac{\mathrm{d}_{\mathrm{j}}}{2} \sin (t)
\end{array}\right.
$$

where $d_{j}$ is the diameter of the jet and, in this case, is equal to the nozzle diameter $\left(d_{n}\right)$.

The envelope of such family of circumferences is:

$$
\left\{\begin{array}{l}
\lambda=\frac{1}{2 \pi \mathrm{f}_{\mathrm{v}}}\left(\arcsin \left(\frac{d_{j} u}{2 \pi f_{\mathrm{f}} A} \cos (t)\right)+t\right) \\
\mathrm{x}=\mathrm{A} \cdot \cos \left(2 \pi \cdot \mathrm{f}_{\mathrm{v}} \cdot \lambda\right)+\mathrm{u} \cdot \lambda+\frac{\mathrm{d}_{\mathrm{j}}}{2} \cos (t) \\
\mathrm{y}=\mathrm{A} \cdot \sin \left(2 \pi \cdot \mathrm{f}_{\mathrm{v}} \cdot \lambda\right)
\end{array}\right.
$$


In order to simplify the discussion, from the graphical point of view, the envelope of the circumferences, whose centre is in the form of epicycloid, approaches to a form of a sinusoid (Figure 10).

\section{Model validation}

\subsection{Model validation: experimental procedure}

In order to validate the proposed model, the experimental procedure is made up of two stages:

- theoretical estimation of the striation spatial period $\left(X=u / f_{v}\right)$ and the striation height $\left(\mathrm{R}_{\mathrm{w}}\right)$, at fixed operative conditions,

- comparison of the theoretical values (obtained at the end of the first stage) with the measured values on the cut surfaces.

The model validation is realised by two distinguished phases:

- measurement of the frequency and the amplitude of the jet oscillation (through the indirect measure of the cutting head vibrations) in order to estimate theoretically the striation dimensional characteristics, using the proposed model. In accordance with the experimental results, presented in paragraph 4.2 , it has been assumed that the cutting head vibration has a single harmonics at $20 \mathrm{~Hz}$ with amplitude of $35 \mu \mathrm{m}$;

- measurement of the roughness profile of the obtained surface in order to confirm the validity of the model. The tests have been carried out on plasticine and it has been measured, through a contact roughness measuring machine, after a suitable freezing treatment, the surface profiles at $1 \mathrm{~mm}$ from the top edge. In order to measure only the effects of the endogenous vibrations, the exogenous vibrations are out of 
consideration in this case, the test piece was fixed to an equipment, completely isolated from the cutting table.

Since the mean striation between the striation edges depends on the feed rate, the validation has been carried out with two different level of feed rate (1000 and 5000 $\mathrm{mm} / \mathrm{min}$ ) at the same experimental conditions (every test with 10 replications).

\subsection{Model validation: experimental results}

As regards the striation spatial frequency $(1 / \mathrm{X})$, the model explains very well the phenomenon; as a function of the feed rate, the predicted mean spatial between the edges of the striations coincides with that measured on the obtained WJ cutting surfaces (Table 2, Figure 11).

The proposed model estimates the amplitude $\left(\mathrm{R}_{\mathrm{w}}\right)$ of the surface irregularities (striations) too. Therefore, the theoretical value predicted by the model $R_{\mathrm{w}}{ }^{*}(20)$, assuming that the cutting head vibration has a single harmonics at $20 \mathrm{~Hz}$, has been compared with the striation amplitude measured on the surfaces. Particularly, the roughness index $\mathrm{P}_{t}$ has been considered because it is the best in order to represent roughness variations on the measured surface. Figure 12 shows the $\mathrm{P}_{\mathrm{t}}$ trend for ten replications for two values of feed rate; table 3 represents the mean and the variance of these values.

For both values of feed rate, at a vibration frequency of $20 \mathrm{~Hz}$, the amplitude of the striations $R_{\mathrm{w}}{ }^{*}(20)$ is equal to two times the value of the vibration amplitude, that is $70 \mu \mathrm{m}$. 
The experiment results indicate that the striation mean amplitude is independent of the feed rate and therefore confirm the uniqueness of $R_{\mathrm{w}}{ }^{*}(20)$ estimated by the model. However, the estimated value diverges from that measured.

The real motion of the vibrating jet is epicycloidal; therefore, the trajectory amplitude of the jet depends on the measured depth. The proposed geometric model, instead, considers the striation amplitude independent of the depth. In fact, the tests have been carried out with a stand off distance of $2 \mathrm{~mm}$ and roughness measurements are done at a distance of $1 \mathrm{~mm}$ from the upper edge. Therefore the radius of the epicycloid, at a particular distance from the nozzle, can be different from the theoretical value measured on the cutting head.

\section{Conclusions}

The WJ cutting quality depends strongly on the exogenous and endogenous vibrations. A considerable reduction of these vibrations allows to obtain remarkable improvements on the surface quality, especially as regards the striation morphology. In particular, dampened splash back has effects on the roughness surface: the roughness synthetic indices indicate a considerable improvement.

A decrease on the exogenous vibrations is achievable using simple devices: anchorage of the workpiece-grates to support bars, fixing of the workpiece using suitable equipment, grill in order to dampen the splash-back, protection systems of the jet against the sprinklings of water.

The endogenous vibrations have been analyzed too and a simple model has been developed in order to predict the striation dimensions as a function of the cutting head vibration. 
The proposed geometric model has been completely validated with regard to the mean spatial of the striation. Particularly, it has been found that the mean spatial of the striation depends strongly on the side vibration frequency of the jet. The main frequency, that characterizes the vibration signal, has been found on the power spectrum of the surfaces profiles of plasticine, for the two levels of feed rate. At a fixed depth of measurement, the feed rate has any effects on the striation amplitude: this result has been validated by experimental dates.

\section{Nomenclature}

$\begin{array}{ll}\mathrm{P} & \text { Water pressure [MPa] } \\ \mathrm{d}_{\mathrm{n}} & \text { Nozzle diameter }[\mathrm{mm}] \\ \mathrm{u} & \text { Feed rate }[\mathrm{mm} / \mathrm{min}] \\ \mathrm{z} & \text { Stand off distance }[\mathrm{mm}] \\ {[\mathrm{M}]} & \text { Power spectral density } \\ \mathrm{R}_{\mathrm{w}} & \text { Surface striation amplitude } \\ \mathrm{X} & \text { Striation spatial period } \\ \xi & \text { Corrective geometric coefficient }\end{array}$

\section{$\underline{\text { Acknowledgements }}$}

The authors are grateful to UE and MIUR (Italian Ministry of Education, University and Research) for the financial support and Tecnocut for the utilisation of their cutting system. 


\section{$\underline{\text { References }}$}

[1] D. Arola, M. Ramulu, Mechanism of material removal in abrasive waterjet machining, $7^{\text {th }}$ American Water Jet Conference Seattle, Washington, 28-31 August, 1993, pp. 46-64

[2] M. Hashish, Characteristic of surfaces machined with abrasive-waterjets, Journal of Engineering Materials and Technology 113 (1991) 354-362

[3] W. Koenig, C. Wulf, The influence of the cutting parameters on jet forces and the geometry of the kerf, $7^{\text {th }}$ International Symposium on Jet Cutting Technology, 26-28 June, 1984, pp. 179-191

[4] D.K.M. Tan, A model for the surface finish in abrasive-waterjet cutting, $8^{\text {th }}$ International Symposium on Jet Cutting Technology Durham, England, 9-11 Sept., 1986, pp. 309-313

[5] R. J. Wilkins, E. E. Graham, An erosion model for waterjet cutting, Journal of Engineering for Industry 115 (1993)

[6] M. Hashish, Prediction models for AWJ machining operations, $7^{\text {th }}$ American Water Jet Conference Seattle, Washington, 28-31 August, 1993, pp.205-216

[7] M. Hashish, On the modelling of surface waviness produced by abrasive-waterjets, $11^{\text {th }}$ International Symposium on Jet Cutting Technology St.Andrews, Scotland, 8-10 Sept., 1992, pp. $17-34$

[8] N.S. Guo, H. Louis, G. Meier, Surface structure and kerf geometry in abrasive waterjet cutting: formation and optimization, $7^{\text {th }}$ American Water Jet Conference Seattle, Washington, 28-31 August, 1993, pp. 1-25

[9] J. Chao, E.S. Geskin, Y. Chung, Investigation of the dynamics of the surfaces topografy formation during abrasive waterjet machining, $11^{\text {th }}$ International Symposium on Jet Cutting Technology St.Andrews, Scotland, 8-10 Sept., 1992, pp. 593-603

[10] J. Chao, E.S. Geskin, Experimental study of the striation formation and spectral analysis of the abrasive waterjet generated surfaces, $7^{\text {th }}$ American Water Jet Conference Seattle, Washington, 28-31 August, 1993, pp. 27-41

[11] L. Ohlson, J. Powell, C. Magnusson, Mechanisms of striation formation in Abrasive Water jet cutting, $12^{\text {th }}$ International Symposium on Jet Cutting Technology Rouen, France, 25-27 Oct., 1994, pp. 151-164 
[12] F. L. Chen, E. Siores, The effect of cutting jet variation on surface striation formation in abrasive water jet cutting, Journal of Materials Processing Technology 135 (2003) 1-5

[13] J. Chao, G. Zhou, M. C. Leu, E. Geskin, Characteristics of abrasive waterjet generated surfaces and effects of cutting parameters and structure vibration, Transaction of the ASME 117 (1995)

[14] E. Siores, L. Chen, W. C. K. Wong, R. Begg, S. Brandellero, D. Boundy, Improving surface finishing generated by the abrasive waterjet process, Advances in Abrasive Technology, 1997, pp. 187-191 